\title{
A Young Patient with Horizontal Semicircular Cupulolithiasis with Immediate Response to the Therapeutic Head-Shaking Maneuver (HSM)
}

\author{
Ajay Kumar Vats ${ }^{1, \odot}$ \\ ${ }^{1}$ Department of Medicine and Neurology, Chaudhary Hospital and \\ Medical Research Centre Private Limited, Udaipur, Rajasthan, India
}

Address for correspondence Ajay Kumar Vats, MBBS, MD (Medicine), DM (Neurology), FRCP, Chaudhary Hospital and Medical Research Centre Private Limited, 472-473, Sector 4, Hiran Magri, Udaipur, Rajasthan, 313002, India (e-mail: vatsneuro@gmail.com).

\begin{abstract}
Keywords

- cupulolithiasis

- supine roll test

- apogeotropic

- head-shaking maneuver

The apogeotropic variant of horizontal semicircular canal benign paroxysmal positional vertigo is attributed to either short anterior ampullary arm canalolithiasis or to the cupulolithiasis with the otoconial debris adhering to the cupula on utricular (Cup-U) or canal side (Cup-C), rendering it heavy and gravity sensitive. The treatment options for horizontal semicircular cupulolithiasis are not very well defined. A 25 -year-old female patient with 8 days history of vertigo on rolling to either of the lateral recumbent position presented in the first week of November 2019. The supine roll test (SRT) on yawing head maximally to the right as well as to the left elicited an apogeotropic horizontal positional nystagmus lasting more than 1 minute, which was stronger on the right side and indicated a diagnosis of left horizontal semicircular cupulolithiasis. Two short-term follow-ups at 1 and 24 hours after head-shaking maneuver (HSM), with verifying SRT, were undertaken. During either of the two follow-ups, neither the previously elicited horizontal positional nystagmus was observed, nor did the patient have vertigo on rolling to lateral recumbent positions. She was telephonically questioned weekly regarding the recurrence of rotational vertigo for the next 4 weeks, and it was confirmed that she remained symptom free till then. The therapeutic HSM, owing to the inertial forces generated, can detach the otoconial debris from the cupula that renders it heavy and gravity sensitive. Successful offloading of cupula by HSM brings immediate cure in the Cup-U variant of the horizontal semicircular cupulolithiasis.
\end{abstract}

\section{Introduction}

Benign paroxysmal positional vertigo (BPPV) is a common mechanical disorder of the vestibular labyrinth. Horizontal semicircular canal variant of the BPPV (HSC-BPPV) is characterized by positional vertigo and direction-changing horizontal nystagmus inducible by lateral head roll to either side with head anteflexed 30 degrees in the supine position during

Published online September 28, 2021
DOI https://doi.org/ $10.1055 / \mathrm{s}-0041-1736344$ ISSN 2581-9607 the supine roll test (SRT) or head yaw test. - Table 1 shows that 1.94 to $38 \%$ of all BPPV patients diagnosed at any specialty clinic suffer from HSC-BPPV. ${ }^{1-11}$ Evidently, HSC-BPPV is less common than the posterior semicircular canal BPPV.

The HSC-BPPV is caused by the otoconial debris either free-floating within the arms, commonly long posterior (nonampullary) arm and less commonly short anterior (ampullary) arm of the HSC (canalolithiasis), or else becoming

(C) 2021. Indian Society of Otology.

This is an open access article published by Thieme under the terms of the Creative Commons Attribution-NonDerivative-NonCommercial-License, permitting copying and reproduction so long as the original work is given appropriate credit. Contents may not be used for commercial purposes, or adapted, remixed, transformed or built upon. (https://creativecommons.org/licenses/by-nc-nd/4.0/).

Thieme Medical and Scientific Publishers Pvt. Ltd. A-12, 2nd Floor, Sector 2, Noida-201301 UP, India 
Table 1 Frequency of different variants of benign paroxysmal positional vertigo attending a specialty clinic

\begin{tabular}{|l|l|l|l|l|l|}
\hline Authors & No. of patients & PSC-BPPV & HSC-BPPV & ASC-BPPV & Multiple canals \\
\hline De la Meilleure et al, ${ }^{1} 1996$ & 287 & $78.05 \%$ & $16.38 \%$ & - & $5.57 \%$ \\
\hline Honrubia et al, ${ }^{2} 1999$ & 292 & $85.62 \%$ & $5.14 \%$ & $1.37 \%$ & $7.87 \%$ \\
\hline Macias et al, ${ }^{3} 2000$ & 259 & $93.02 \%$ & $1.94 \%$ & - & $5.04 \%$ \\
\hline Korres et al, ${ }^{4} 2002$ & 122 & $90.16 \%$ & $8.2 \%$ & $1.64 \%$ & - \\
\hline Sakaida et al, ${ }^{5} 2003$ & 50 & $56 \%$ & $38 \%$ & & $6 \%$ \\
\hline Imai et al, ${ }^{6} 2005$ & 108 & $64.82 \%$ & $33.33 \%$ & - & $1.85 \%$ \\
\hline Nakayama and Epley, ${ }^{7} 2005$ & 833 & $66.39 \%$ & $10.08 \%$ & $2.28 \%$ & $21.25 \%$ \\
\hline Cakir et al, ${ }^{8} 2006$ & 169 & $85.21 \%$ & $11.83 \%$ & $1.18 \%$ & $1.78 \%$ \\
\hline Moon et al, ${ }^{9} 2006$ & 1,692 & $60.9 \%$ & $31.9 \%$ & $2.2 \%$ & $5.0 \%$ \\
\hline Jackson et al, ${ }^{10} 2007$ & 260 & $66.9 \%$ & $11.9 \%$ & $21.2 \%$ & - \\
\hline Chung et al, ${ }^{11} 2009$ & 589 & $61.8 \%$ & $35.3 \%$ & $2.9 \%$ & - \\
\hline
\end{tabular}

Abbreviations: ASC-BPPV, anterior semicircular canal benign paroxysmal positional vertigo; HSC-BPPV, horizontal semicircular canal benign paroxysmal positional vertigo; PSC-BPPV, posterior semicircular canal benign paroxysmal positional vertigo.

adherent to the cupula (cupulolithiasis) on canal (Cup-C) or utricular side (Cup-U). ${ }^{12}$

Because three different possible sites of pathologies within the HSC can masquerade as apogeotropic HSC-BPPV (short anterior ampullary arm canalolithiasis, Cup-C and Cup-U types of cupulolithiasis), it is imperative to unerringly localize one of the three pathological sites responsibly leading to its causation. If the SRT elicits persistent apogeotropic horizontal nystagmus lasting $\geq 1$ minute and there are no changes in the direction of nystagmus even after repetitive head roll tests, it is explicable by the horizontal canal cupulolithiasis either on canal-side (Cup-C) or on the utricular-side (Cup-U). ${ }^{13}$ The side to which otoconial debris is adherent (Cup-C or Cup-U) in the horizontal semicircular cupulolithiasis cannot be determined except perhaps by response to treatment in selected cases.

The treatment options for the horizontal semicircular cupulolithiasis are not very well established. The wide variety of methods described in the medical literature for treating horizontal semicircular cupulolithiasis namely forced prolonged positioning, ${ }^{14}$ head-shaking maneuver (HSM) ${ }_{15,16}$ mastoid vibration, ${ }^{17}$ and cupulolith repositioning maneuvers (CuRM) target to detach otoconial debris adherent to the utricular (Cup-U) and/or canal (Cup-C) side of the cupula. ${ }^{18,19}$ A recent double-blind randomized controlled trial comparing efficacy of CuRM, HSM, and modified Lempert maneuver found that the CuRM is theoretically a better therapeutic option, but the therapeutic efficacy of CuRM was not statistically different compared with the other two maneuvers on the 2 nd day and at 1 week after treatment. ${ }^{20}$

A case of a 25-year-old female patient that presented with an 8-day history of vertigo on rolling to either of the lateral recumbent positions is reported. Her SRT elicited an asymmetrical apogeotropic horizontal positional nystagmus (right stronger than left) lasting $\geq 1$ minute on lateral head roll to either side. She was successfully treated with therapeutic HSM with the head pitched 30 degrees in flexion. At 1 hour and after 24 hours after the HSM, she neither had vertigo on rolling to either of the lateral recumbent positions nor the SRT elicited horizontal positional nystagmus. The author was able to video record the SRT eliciting apogeotropic horizontal positional nystagmus before treatment and its disappearance at 1 hour and after 24 hours.

\section{Case Description}

\section{History}

A 25-year-old female patient presented in the first week of November 2019 with 8 days history of vertigo on rolling to either of the lateral recumbent positions. There was no history of staggering during the walk, diplopia, difficulty in swallowing, hiccups, drooping of upper eyelids, dysarthria, or facial or limb weakness. There was also no history of coronary artery disease, diabetes, hypertension, hypothyroidism, jaundice, craniocervical trauma, cervical radiculopathies, cervical canal stenosis, rheumatoid arthritis, Paget's disease, ankylosing spondylitis, low back dysfunction, spinal cord injuries, or cerebrovascular disease.

\section{Examination}

The general physical examination and vitals of the patient were normal. The screening examination of the cervical spine did not reveal any limitation of movement. The examination of the back region did not reveal kyphoscoliosis. The examination of the lumbosacral spine, including straight-leg raising (SLR) test and reverse SLR test, was normal. The neurological examination revealed normal cranial nerve examination; strength was grade $5 / 5$ in all four limbs with normal deep tendon reflexes, and bilateral plantar reflexes were flexor. The examination of the cerebellar system revealed no spontaneous or gaze-evoked nystagmus, and there was no appendicular or axial incoordination. The otoneurological examination revealed normal vertical and horizontal saccadic and smooth pursuit eye movements. The head impulse test was bilaterally normal. The presence of spontaneous nystagmus was ruled out by using takeaway 


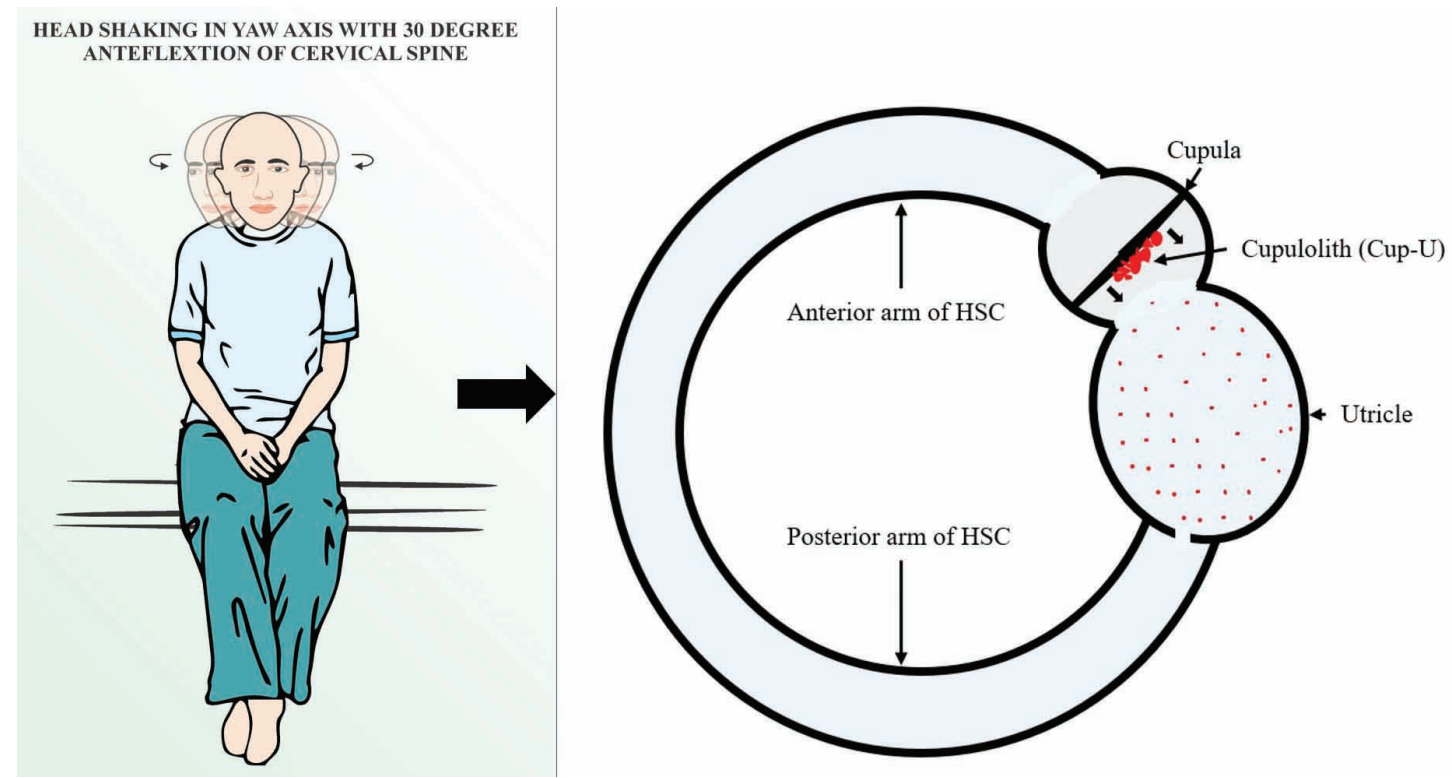

Fig. 1 Head-shaking maneuver (HSM): The patient instructed to be in short-sitting with lower limbs hanging on the long edge of the examination table. The head was anteflexed 30 degrees in the pitch plane and briskly shaken by the excursions of 30 degrees side-to-side at an approximate rate of $3 \mathrm{~Hz}$ in the yaw axis for around 25 seconds (right panel). Owing to the inertial force generated by rapid acceleration and deceleration during HSM, the otoconial debris adherent to the utricular side of the cupula of horizontal semicircular canal (in red) detaches and disperses in the utricular matrix (small red dots).

Frenzel goggles. ${ }^{21}$ The Dix-Hallpike test performed, as per the clinical practice guidelines, of the American Academy of Otolaryngology, Head, and Neck Surgery Foundation, ${ }^{22}$ did not elicit positional nystagmus in the 20 degrees head hanging position to the either side. The SRT was performed with the patient in long sitting on the examination table. She was made to lay supine with her head landing on a four-inch-thick pillow, so it got anteflexed to 30 degrees in this position. SRT elicited an apogeotropic horizontal positional nystagmus on yawing the head maximally to the right as well as to the left but was visibly stronger on the right. The latency of the apogeotropic horizontal positional nystagmus was 4 seconds on either side, and its duration is 190 seconds on the right and 60 seconds on the left side (-Video 1). The SRT indicated the involvement of the left HSC. The characteristics of the apogeotropic horizontal positional nystagmus did not change during several cycles of the diagnostic SRT, and lasted $\geq 1$ minute, implying in all probability a pathology of left horizontal semicircular cupulolithiasis either to the canal (Cup-C) or utricular side (Cup-U).

\section{Video 1}

Supine roll test elicits an apogeotropic horizontal positional nystagmus on yawing the head maximally to the right as well as to the left, which is visibly stronger on the right. The latency of the apogeotropic horizontal positional nystagmus is 4 seconds on either side, and its duration is 190 seconds on the right and 60 seconds on the left side.
The characteristics of the apogeotropic horizontal positional nystagmus did not change during several cycles of the diagnostic supine roll test, implying in all probability a pathology of left horizontal semicircular cupulolithiasis. Online content including video sequences viewable at: https://www.thieme-connect.com/products/ejournals/ html/10.1055-s-0041-1736344-aono-20-9-0110-v1.

\section{Diagnosis}

The aforesaid clinical history and examination, is consistent with the diagnosis of left horizontal semicircular BPPV (apogeotropic variant, possibly, left HSC cupulolithiasis). Video recording of the initial SRT ( - Video 1 ), not merely facilitated the identification of apogeotropic positional nystagmus (and hence localization of the involved semicircular canal) but also precisely lateralized the involved HSC by comparing its strength, as per the Ewald's second law. The video recording of the SRT that elicited asymmetric apogeotropic horizontal positional nystagmus was observed several times on a bigger screen of the computer to identify the weaker nystagmus, and hence the involved HSC.

\section{Intervention}

HSM ( - Fig. 1, - Video 2) was performed with the patient in short-sitting and lower limbs hanging on the long edge of the examination table. The head was anteflexed 30 degrees in the pitch plane and briskly shaken by the excursions of 30 degrees side-to-side at an approximate rate of $3 \mathrm{~Hz}$ in the yaw axis for around 25 seconds. Two sequent HSM were done in one session of treatment. 


\section{Video 2}

Head-shaking maneuver (HSM) is performed with the patient in short-sitting and lower limbs hanging along the long edge of the examination table. The head is anteflexed 30 degrees in the pitch plane and briskly shaken by the excursions of 30 degrees side-to-side at an approximate rate of $3 \mathrm{~Hz}$ in the yaw axis for around 30 seconds. Online content including video sequences viewable at: https://www.thieme-connect.com/products/ejournals/ html/10.1055-s-0041-1736344-aono-20-9-0110-v2.

\section{Prognosis and Intervention}

An SRT done at 1 hour ( - Video 3 ), and repeated 24 hours after ( - Video 4 ), the therapeutic HSM did not elicit any positional nystagmus. The patient neither complained of rotational vertigo or any nonvertiginous dizziness either after 1 hour or 24 hours after the therapeutic HSM indicating cure. She was telephonically questioned weekly regarding the recurrence of rotational vertigo for the next 4 weeks, and it was confirmed that she remained symptom free till then.

\section{Video 3}

The verifying supine roll test of patient performed an hour after the head-shaking maneuver (HSM) did not elicit any lying down nystagmus or horizontal positional nystagmus on maximal yawing of the head to the right and left and the patient did not complain of vertigo either. Online content including video sequences viewable at: https://www.thieme-connect.com/products/ejournals/ html/10.1055-s-0041-1736344-aono-20-9-0110-v3.

\section{Video 4}

The verifying supine roll test of patient performed 24 hours after the head-shaking maneuver (HSM) did not elicit any lying down nystagmus or horizontal positional nystagmus on maximal yawing of the head to the right and left and the patient did not complain of vertigo either. Online content including video sequences viewable at: https://www.thieme-connect.com/products/ejournals/ html/10.1055-s-0041-1736344-aono-20-9-0110-v4.

\section{Discussion}

The apogeotropic variant of HSC-BPPV is caused by either short anterior (ampullary) arm canalolithiasis due to free-floating otoconial debris or by the latter getting adherent to the cupula rendering it heavy and gravity sensitive (cupulolithiasis). The otoconial debris can adhere to either canal (Cup-C) or utricular side (Cup-U) of the cupula. The treatment options for the horizontal semicircular cupulolithiasis are not very established. HSM can result in immediate resolution of positional vertigo and nystagmus if the otoconial debris is adherent to the utricular side of the cupula (Cup-U). On the contrary, in the Cup-C variant of the horizontal semicircular cupulolithiasis, the otoconial debris getting detached by HSM is relocated in the HSC either in its short anterior (ampullary) arm or the long posterior (nonampullary) arm; thereupon transformation to either geotropic long posterior (nonampullary) arm horizontal semicircular canalolithiasis or the apogeotropic short anterior ampullary arm canalolithiasis occurs.

The rapid acceleration and deceleration during HSM generate inertial forces in the otoconial debris adherent to the cupula that causes its detachment. In the case reported here, two short-term follow-ups at 1 hour and 24 hours after HSM, with verifying SRT, were undertaken. In either of the two follow-ups, neither the previously elicited horizontal positional nystagmus was observed, nor did the patient have vertigo on rolling to lateral recumbent positions. Given the high rate of spontaneous resolution in HSC-BPPV reported in some studies, ${ }^{6,23}$ a favorable therapeutic audit of HSM at two short-term follow-ups indicate a beneficial effect of applied maneuver rather than spontaneous remission. The dramatic response of HSM in the reported case indicates that the patient suffered from Cup-U variant of cupulolithiasis. If the patient had Cup-C variant of HSC-BPPV, the otoconial debris disengaged by the inertial forces generated during HSM would have fallen into the canal thereby transforming it to the left horizontal semicircular canalolithiasis (apogeotropic/geotropic).

\section{Conclusion}

Patients with a history of vertigo triggered by positional changes of rolling on the bed that elicits apogeotropic horizontal positional nystagmus of $\geq 1$ minute duration on SRT, which does not change after many sequences of SRT, in all probability suffer from the horizontal semicircular cupulolithiasis. An HSM is an excellent option to offload the heavy cupula by generating inertial forces by rapid acceleration and deceleration of the head in the yaw axis. If HSM disengages the otoconial debris from the cupula, it either results in a cure in Cup-U variant; and in the Cup-C variant, it is liable to transform cupulolithiasis of the HSC into canalolithiasis (geotropic or apogeotropic), which is much more amenable to the repositioning maneuvers.

\section{Declaration of Interest}

The author has no disclosures to report.

\section{Disclaimers}

The views expressed in the submitted article are author's own and not an official position of the institution to which author is affiliated. 


\section{Conflict of Interest}

The author whose name is listed above certifies that he has no affiliations with or involvement in any organization or entity with any financial interest (such as honoraria; educational grants; participation in speakers' bureaus; membership, employment, consultancies, stock ownership, or other equity interest; and expert testimony or patent-licensing arrangements), or nonfinancial interest (such as personal or professional relationships, affiliations, knowledge or beliefs) in the subject matter or materials discussed in this manuscript.

\section{Acknowledgment}

Renith Kurian video recorded the diagnostic and therapeutic maneuvers and precisely captured the nystagmus during the entire diagnostic and treatment period.

\section{References}

1 De la Meilleure G, Dehaene I, Depondt M, Damman W, Crevits L, Vanhooren G. BPPV of the horizontal canal. J Neurol Neurosurg Psychiatry 1996;60(1):68-71

2 Honrubia V, Baloh RW, Harris MR, Jacobson KM. Paroxysmal positional vertigo syndrome. Am J Otol 1999;20(4):465-470

3 Macias JD, Lambert KM, Massingale S, Ellensohn A, Fritz JA. Variables affecting treatment in BPPV. Laryngoscope 2000;110(11):1921-1924

4 Korres S, Balatsouras DG, Kaberos A, Economou C, Kandiloros D, Ferekidis E. Occurrence of semicircular canal involvement in BPPV. Otol Neurotol 2002;23(6):926-932

5 Sakaida M, Takeuchi K, Ishinaga H, Adachi M, Majima Y. Long-term outcome of BPPV. Neurology 2003;60(9):1532-1534

6 Imai T, Ito M, Takeda N, et al. Natural course of the remission of vertigo in patients with benign paroxysmal positional vertigo. Neurology 2005;64(5):920-921

7 Nakayama M, Epley JM. BPPV and variants: improved treatment results with automated, nystagmus-based repositioning. Otolaryngol Head Neck Surg 2005;133(1):107-112

8 Cakir BO, Ercan I, Cakir ZA, Civelek S, Sayin I, Turgut S. What is the true incidence of horizontal semicircular canal BPPV. Otolaryngol Head Neck Surg 2006;134(3):451-454

9 Moon SY, Kim JS, Kim BK, et al. Clinical characteristics of BPPV in Korea: a multicenter study. J Korean Med Sci 2006;21(3):539-543
10 Jackson LE, Morgan B, Fletcher JC, Krueger WW. Anterior canal BPPV: an underappreciated entity. Otol Neurotol 2007;28(2):218-222

11 Chung KW, Park KN, Ko MH, et al. Incidence of horizontal canal BPPV as a function of the duration of symptoms. Otol Neurotol 2009;30(2):202-205

12 Bruintjes TD, Masius-Olthof S, Kingma H. Benign paroxysmal positional vertigo of the horizontal canal. Clin of Otorhinolaryngology 2017;1(1):7-14

13 von Brevern M, Bertholon $\mathrm{P}$, Brandt $\mathrm{T}$, et al. Benign paroxysmal positional vertigo: Diagnostic criteria. J Vestib Res 2015;25(3-4):105-117

14 Chiou WY, Lee HL, Tsai SC, Yu TH, Lee XX. A single therapy for all subtypes of horizontal canal positional vertigo. Laryngoscope 2005;115(8):1432-1435

15 Vannucchi P, Giannoni B, Nuti D, La cupololitiasi del canale semicircolare orizzontale: Aspetti clinici. In: Pagnini P, ed. La cupololitiasi. Milan, Italy: Formenti Puropharma; 1992 81-91

16 Kim JS, Oh SY, Lee SH, et al. Randomized clinical trial for apogeotropic horizontal canal benign paroxysmal positional vertigo. Neurology 2012;78(3):159-166

17 Kim HA, Park SW, Kim J, et al. Efficacy of mastoid oscillation and the Gufoni maneuver for treating apogeotropic horizontal benign positional vertigo: a randomized controlled study. J Neurol 2017;264(5):848-855

18 Zuma e Maia F. New treatment strategy for apogeotropic horizontal canal benign paroxysmal positional vertigo. Audiology Res 2016;6(2):163

19 Kim SH, Jo SW, Chung WK, Byeon HK, Lee WS. A cupulolith repositioning maneuver in the treatment of horizontal canal cupulolithiasis. Auris Nasus Larynx 2012;39(2):163-168

20 Kong TH, Song MH, Kang JW, Shim DB. Double-blind randomized controlled trial on efficacy of cupulolith repositioning maneuver for treatment of apogeotropic horizontal canal benign paroxysmal positional vertigo. Acta Otolaryngol 2020;140(6):473-478

21 Strupp M, Fischer C, Hanß L, Bayer O. The takeaway Frenzel goggles: a Fresnel-based device. Neurology 2014; 83(14):1241-1245

22 Bhattacharyya N, Gubbels SP, Schwartz SR, et al. Clinical practice guideline: benign paroxysmal positional vertigo (update. Otolaryngol Head Neck Surg 2017;156(3_suppl) :S1-S47

23 Parnes LS, Agrawal SK, Atlas J. Diagnosis and management of benign paroxysmal positional vertigo (BPPV. CMAJ 2003;169(7):681-693 